\title{
A potential window into surgical outcomes for Berry syndrome
}

\author{
S. Ram Kumar, MD, PhD, FACS
}

\footnotetext{
From the Division of Cardiac Surgery, Department of Surgery, University of Southern California; and the Heart Institute, Children's Hospital of Los Angeles, Los Angeles, Calif.

Disclosures: Author has nothing to disclose with regard to commercial support

Received for publication Jan 30, 2017; accepted for publication Jan 30, 2017; available ahead of print March 6, 2017.

Address for reprints: S. Ram Kumar, MD, PhD, FACS, Children's Hospital, Los Angeles, 4650 Sunset Blvd, Mailstop 66, Los Angeles, CA 90027 (E-mail: rsubramanyan@chla.usc.edu).

J Thorac Cardiovasc Surg 2017;153:1148-9

$0022-5223 / \$ 36.00$

Copyright (c) 2017 by The American Association for Thoracic Surgery

http://dx.doi.org/10.1016/j.jtcvs.2017.01.041
}

During embryogenesis, cardiac progenitor cells that originate within the pharyngeal mesoderm (loosely termed second heart field cells) integrate into the developing heart and contribute to the formation of the right ventricle, the outflow tracts, the main pulmonary artery (PA), and the aortic arch. Intricate spatially and temporally regulated molecular events orchestrate the appropriate division and maturation of the primitive outflow tract, usually resulting in the normal postnatal anatomy. The complexity in this process is manifested by the fact that conotruncal outflow tract defects comprise nearly $30 \%$ of all congenital heart defects. Because of the close relationship between the different maturation events in the outflow tract, it is common for multiple developmental defects in this region of the heart to coexist. In 1982, Berry and colleagues ${ }^{1}$ described an interesting array of associated defects characterized by a distal aortopulmonary window, aortic origin of the right PA, intact ventricular septum, and interruption or coarctation of the aortic isthmus with a patent ductus arteriosus. This group of defects has since been called the Berry syndrome. The report of Berry and colleagues ${ }^{1}$ elegantly discussed the embryogenesis of this complex abnormality. A distal aortopulmonary septal defect results in failure of complete septation of the distal outflow tract, resulting in an aortopulmonary window. In addition, failure of the sixth pharyngeal arch arteries to fuse with each other and the developing main pulmonary trunk results in wide separation of the right and left PAs, with the resulting aortic origin of the right PA (Figure 1). In their article in this issue of the Journal, Hu and colleagues ${ }^{2}$ propose a hemodynamic etiology for the arch abnormality, indicating that the siphoning of blood from the ascending aorta into the right PA and resultant reduced flow into the aortic isthmus is responsible for arch hypoplasia, or interruption in its most extreme form. Since the original report of Berry and colleagues, ${ }^{1}$ several small series and individual case reports have documented successful surgical management of Berry syndrome. The article by $\mathrm{Hu}$ and colleagues ${ }^{2}$ is a report of surgical outcomes in 16 consecutive infants with Berry

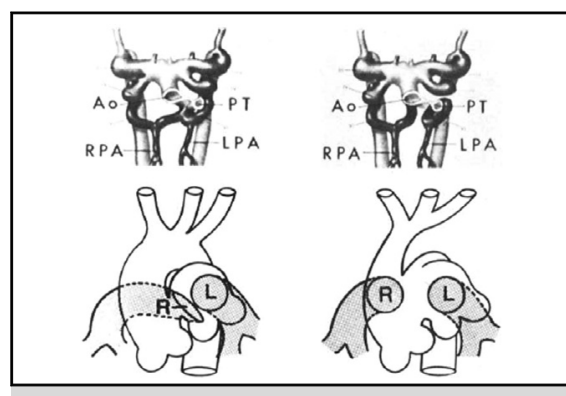

Embryologic events believed to result in corresponding types of aortopulmonary window.

\section{Central Message}

Outcomes for single-stage repair of Berry syndrome continue to improve as focus shifts to limiting the need for long-term reintervention.

See Article page 1139 .

syndrome, comprising the largest patient cohort reported to date. $\mathrm{Hu}$ and colleagues ${ }^{2}$ document commendable short-term and midterm results of single-stage repair, with $13 \%$ surgical mortality and $75 \%$ freedom from reintervention at a mean 5 years of follow-up. Surviving patients have excellent anatomic and physiologic profiles.

Interestingly, only 4 patients in this series underwent repair as neonates, and half of the patients were older than 3 months at surgery, which probably reflects an institutional referral bias. What is interesting is that, despite such a delayed presentation, the patent ductus arteriosus was able to maintain adequate systemic perfusion to the lower half of the body such that no patient was placed on prostaglandin therapy. In addition, it is well known that the pulmonary vasculature is at high risk of development of early and severe hypertension in this disease process. Even so, fewer than a third of patients in this series presented with cyanosis, and there was no evidence of advanced pulmonary vascular changes in any patient. After the early recovery period, $43 \%$ patients required pulmonary vasodilator therapy, but only for a very short time thereafter. Such outcomes are better than what one would predict on the basis of the pathophysiology of this lesion. The most plausible explanation lies in the limitation that this is a retrospective analysis of only those patients who presented for surgical management. It is entirely possible that some specific aspect of these patients' anatomy led to a better balance between systemic and pulmonary circulations, such that there was a selection 



FIGURE 1. Artist's renderings (top panels) and diagrammatic illustrations (bottom panels) of embryologic events believed to result in the anatomic entities of isolated aortopulmonary window (left panels) and Berry syndrome (right panels). Ao, Aorta; PT, pulmonary trunk; RPA, right pulmonary artery; $L P A$, left pulmonary artery. Reprinted with permission. ${ }^{1}$

of patients with prolonged survival without intervention. An unknown subset of potentially sicker patients who lacked such an advantage probably died early without ever being offered repair. The question then remains whether such impressive results can be reproduced when all patients are appropriately referred and operated on early in the neonatal period. This concern is particularly relevant when one considers that 2 of the 3 patients who died in this series were neonates, representing $50 \%$ mortality in the neonatal subgroup.

As is the case in the vast majority of congenital heart diseases, immediate surgical outcomes for single-stage repair of Berry syndrome continue to improve at a rapid pace. The focus is now shifting to long-term outcomes, with a particular stress on the need for surgical reintervention. $\mathrm{Hu}$ and colleagues ${ }^{2}$ report 3 PA reinterventions and 1 aortic reintervention. It is conceivable that the need for PA reintervention is related to technique, as has been suggested by $\mathrm{Hu}$ and colleagues, ${ }^{2}$ but this cannot be conclusively demonstrated because of the small number of patients. It is also possible that other technical alterations not used by $\mathrm{Hu}$ and colleagues ${ }^{2}$ may further mitigate the need for reintervention. If the shortened aorta is compressing the right PA, could one more routinely use the LeCompte maneuver? Alternatively, should one extend the ascending aorta with an interposition graft? Should $\mathrm{Hu}$ and colleagues ${ }^{2}$ have primarily used an anterior patch to augment the PA when using the posterior aortic cuff technique? Regardless of these technical nuances, this report is another sober reminder that symmetric and proportional growth of the PA cannot be uniformly expected following surgical intervention early in life. Despite several technical modifications and types of patch materials, the ideal approach to ensure PA growth in this and many other congenital cardiac disease processes continues to be elusive. Advances in engineering biologically compatible conduits and patches that can at the very least keep up with somatic growth continue to be hotly pursued and eagerly anticipated.

\section{References}

1. Berry TE, Bharati S, Muster AJ, Idriss FS, Santucci B, Lev M, et al. Distal aortopulmonary septal defect, aortic origin of the right pulmonary artery, intact ventricular septum, patent ductus arteriosus and hypoplasia of the aortic isthmus: a newly recognized syndrome. Am J Cardiol. 1982;49:108-16.

2. Hu R, Zhang W, Liu X, Dong W, Zhu H, Zhang H. Current outcomes of one-stage surgical correction for Berry syndrome. J Thorac Cardiovasc Surg. 2017; 153: $1139-47$. 